\title{
Motivación y satisfacción laboral del personal de una organización de salud del sector privado
}

\author{
Helen Stephani Marin Samanez ${ }^{1}$, Maritza Dorila Placencia Medina ${ }^{2}$
}

RESUMEN

Objetivo: La motivación y la satisfacción laboral son claves para la Gestión de Recursos Humanos en un enfoque de calidad total. El objetivo del presente estudio es establecer la relación entre la motivación laboral y la satisfacción laboral del personal de Socios en Salud Sucursal Perú; asimismo, determinar el nivel de motivación laboral del personal según la Teoría Bifactorial de Frederick Herzberg y determinar el nivel de satisfacción laboral del personal de acuerdo con las dimensiones del instrumento Font Roja.

Materiales y métodos: Estudio descriptivo, observacional, transversal de tipo relacional. La muestra fue de 136 trabajadores quienes realizaron una encuesta autoaplicada entre los meses de febrero y junio del 2016.

Resultados: El nivel de motivación laboral fue "medianamente motivado" (49.3\%). Respecto a los factores higiénicos, los trabajadores resultaron medianamente motivados (46.3\%), y los factores con mayores promedios globales fueron: "Relaciones con el jefe" y "Relaciones con los compañeros de trabajo", mientras que los de menor promedio fueron: "Prestigio o status" y "Políticas y directrices de la organización". Respecto a los factores motivacionales, los trabajadores resultaron medianamente motivados (57.4\%), y los factores con mayores promedios fueron: "El Trabajo en sí mismo como estímulo positivo" y "Responsabilidad", mientras que el de menor promedio fue: "Desarrollo profesional". El nivel de la satisfacción laboral fue "medianamente satisfecho" (56.6\%), y el componente con mayor promedio global fue "Relación personal", mientras que los componentes con menores promedios globales fueron "Presión en el trabajo", "Variedad de la tarea" y "Distensión en el trabajo". El coeficiente de Spearman fue de 0.336. Se aceptó que "a mayor grado de motivación laboral, mayor grado de satisfacción laboral del personal de Socios en Salud Sucursal Perú".

Conclusiones: La relación entre la motivación y la satisfacción laboral es de una baja correlación positiva. El nivel de la motivación laboral fue "medianamente motivado". El nivel de la satisfacción laboral fue "medianamente satisfecho".

Palabras clave: Motivación; satisfacción en el trabajo; recursos humanos; rendimiento laboral; calidad total; gestión de la calidad (Fuente: DeSC BIREME).

\section{Work motivation and job satisfaction of a private health care organization staff} ABSTRACT

Objective: Work motivation and job satisfaction are key elements for Human Resource Management from a total quality approach. This study aims to establish the relationship between work motivation and job satisfaction of the staff of the Peruvian branch of Partners in Health, to determine the staff's level of work motivation according to Frederick Herzberg's Two-Factor Theory, and to determine the staff's level of job satisfaction according to the dimensions of the Font Roja questionnaire.

Materials and methods: A descriptive, observational, cross-sectional, relational study. The sample consisted of 136 workers who completed a self-administered survey between February and June 2016.

Results: The level of work motivation was "moderately motivated" (49.3\%). Regarding the hygiene factors, workers were moderately motivated (46.3\%), and the factors with the highest overall averages were "Relationship with the boss" and "Relationship with co-workers", while the factors with the lowest averages were "Prestige or status" and "Policies and guidelines of the organization". Regarding the motivational factors, workers were moderately motivated (57.4\%), and the factors with the highest averages were "The job by itself as a positive stimulus" and "Responsibility", while the factor with the lowest average was "Professional development". The level of job satisfaction was "moderately satisfied" (56.6\%), and the component with the highest overall average was "Personal relationship", while the components with the lowest overall averages were "Pressure at work", "Variety of the task" and "Relaxation at work". The Spearman coefficient was 0.336 . The following statement was accepted: "The greater the work motivation, the greater the job satisfaction of the staff of the Peruvian branch of Partners in Health."

Conclusions: The relationship between work motivation and job satisfaction has a low positive correlation. The level of work motivation was "moderately motivated". The level of job satisfaction was "moderately satisfied".

Keywords: Motivation; job satisfaction; human resources; work performance; total quality; quality management (Source: MeSH NLM).

1 Socios en Salud Sucursal Perú, Dirección de Proyectos de Investigación. Lima, Perú.

2 Sección de Farmacología, Departamento Académico de Ciencias Dinámicas, Facultad de Medicina, Universidad Nacional Mayor de San Marcos (UNMSM). Lima, Perú. 


\section{INTRODUCCIÓN}

Socios en Salud Sucursal Perú (SES) es una organización dedicada a la salud y es filial de la organización internacional sin fines de lucro Partners in Health (PIH). SES lleva 20 años trabajando en Perú y actualmente, realiza principalmente, Proyectos de investigación y de intervención en salud que comprenden principalmente temas de tuberculosis, $\mathrm{VIH}$, salud mental, salud materno infantil, entre otros. El personal de la organización está conformado por profesionales de la salud, personal técnico en salud y personal administrativo. En los últimos 3 años, la organización ha pasado por una reorganización administrativa con la que se ha dado mayor importancia a la Gestión de Recursos Humanos ${ }^{(1,2,3)}$.

La motivación laboral es un estado interno que activa y direcciona nuestros pensamientos y está relacionada a todos aquellos factores capaces de provocar, mantener y dirigir la conducta hacia un objetivo y todos ellos generan conductas que varían en el grado de activación o de intensidad del comportamiento ${ }^{(4)}$. Centrándonos en la estructura del comportamiento, los trabajadores aportan de acuerdo a la motivación por sus propios intereses y tratan de imponerlo a la organización a la que pertenecen. Sin embargo, una herramienta esencial para que las instituciones alcancen el éxito, son las personas que la integran. Si la gerencia hace una correcta administración de los recursos puede estimular positivamente el logro de las metas personales y las metas institucionales traducido en un buen rendimiento de los trabajadores ${ }^{(5-8)}$.

Según la Teoría Bifactorial de Frederick Herzberg se puede diferenciar: factores higiénicos (relacionados al ambiente que rodea a las personas y abarcan las condiciones en que desempeñan su trabajo; variables que se encuentran administradas por la empresa como Relaciones con el jefe, Relaciones con compañeros de trabajo, Prestigio o status, Buenas Condiciones Laborales, Políticas y Directrices de la Organización y Seguridad de trabajo) y factores motivacionales (relacionados más directamente a la satisfacción de la persona como El Trabajo en sí mismo como estímulo positivo, Reconocimiento profesional, Desarrollo profesional, Responsabilidad, Oportunidad de Crecimiento y autorrealización personal) ${ }^{(9-12)}$.

La satisfacción laboral en cambio, es un estado emocional positivo que se deriva de la percepción subjetiva de las experiencias laborales de un individuo, marcado por el balance final entre el rol que cada trabajador quiere cumplir y el rol que finalmente desempeña ${ }^{(13-16)}$. El cuestionario FONT Roja es un instrumento utilizado ampliamente en el sector salud que considera 7 componentes para medir la satisfacción laboral: Satisfacción por el trabajo, Presión en el trabajo, Relación personal, Distensión en el trabajo, Adecuación para el trabajo, Control sobre el trabajo y variedad de la tarea.

En el ámbito nacional e internacional se han realizado investigaciones que han permitido describir y analizar la motivación y satisfacción en diversos ámbitos laborales aunque todas hacen uso de cuestionarios diferentes para su medición. Las organizaciones que aplican seriamente la gestión de la calidad, por razones de mejoramiento tienen mejores resultados de rendimiento que las empresas que buscan una certificación ISO por ser requisito de confiabilidad. En el marco de enfoque de calidad total en salud, este estudio es un insumo valioso de información pues podría ayudar a identificar puntos críticos y establecer estrategias que permitan alinear los objetivos individuales a los objetivos institucionales, incrementando el valor económico y social de la organización ${ }^{(17)}$.

La investigación tuvo el objetivo de establecer la relación entre la motivación y la satisfacción laboral del personal de Socios en Salud Sucursal Perú. Asimismo, determinar el nivel de motivación laboral del personal según la Teoría Bifactorial de Frederick Herzberg y determinar el nivel de satisfacción laboral del personal de acuerdo a las dimensiones del instrumento FONT Roja.

La hipótesis de investigación propuesta fue: “A mayor grado de motivación laboral, mayor grado de satisfacción laboral del personal de Socios en Salud Sucursal Perú".

Además se propusieron dos hipótesis específicas:

- Hipótesis específica 1: “A mayor grado de motivación laboral en los factores higiénicos, mayor grado de satisfacción laboral del personal de Socios en Salud Sucursal Perú".

- Hipótesis específica 2: “A mayor grado de motivación laboral en los factores motivacionales, mayor grado de satisfacción laboral del personal de Socios en Salud Sucursal Perú”.

\section{MATERIALES Y MÉTODOS}

\section{Diseño del estudio}

Para probar nuestra hipótesis de investigación, se realizó un estudio descriptivo, transversal, observacional de tipo relacional con trabajadores de las cinco oficinas locales de SES. El estudio se ejecutó entre los meses de febrero y junio del 2016.

\section{Población y muestra}

En enero 2016, se reportó 209 colaboradores que laboraban en las 5 oficinas locales en Lima e Ica (Carabayllo, Chimpu Ocllo, San Martín de Porres, San Borja y Chincha) los mismos que fueron invitados a participar en el estudio. Los criterios de inclusión para participar fueron: ser colaborador SES bajo cualquier tipo de contrato en el momento de aplicación del instrumento y con al menos 3 meses en la organización. La muestra final estuvo conformada por 136 trabajadores y se realizó un muestreo por conglomerados. Cada oficina fue considerada como un conglomerado. 


\section{Recolección de datos}

El proceso de reclutamiento de participantes incluyó un comunicado general para informar sobre el estudio y el inicio de la investigación. Esto se acompañó de coordinaciones en las diferentes oficinas, permitiendo realizar una presentación general de la investigación en la oficina local y posteriormente, obtener el consentimiento informado de los colaboradores. Luego, los firmantes recibieron el instrumento de la investigación para su resolución.

El instrumento tuvo tres secciones: 1) Datos Generales, 2) Cuestionario de Motivación Laboral (Cuestionario de Frederick Herzberg) y 3) Cuestionario de Satisfacción Laboral (Cuestionario Font Roja).

Para procesar los datos se realizó una base de datos de Microsoft Office Excel 2010 (Microsoft, Seattle, WA).

En el cuestionario de Frederick Herzberg, a cada criterio/ factor se le puede asignar un puntaje en una escala de 1 a 5 puntos, donde 1 denota rechazo, 3 denota indiferencia y 5 indica aceptación. El puntaje global mínimo es de 12 puntos y el puntaje global máximo es de 60 puntos.

En el cuestionario de FONT Roja, a cada ítem se le puede asignar un puntaje en una escala de 1 a 5 puntos, donde 1 indica rechazo, 3 indica indiferencia y 5 indica aceptación. El puntaje global mínimo es de 24 puntos y el puntaje global máximo es de 120 puntos. Todos los ítems negativos requirieron de una recodificación ya que por su contenido gramatical se deben calificar de forma inversa.

\section{Análisis estadístico}

Para cada variable, se establecieron los diferentes rangos de puntaje usando una Escala de Stanones.

Las variables de motivación y satisfacción laboral fueron analizadas individualmente: la motivación bajo la Teoría de Frederick Herzberg; mientras que la satisfacción bajo los 7 componentes del cuestionario FONT Roja.

Se buscó establecer la relación entre ambas variables a través de un análisis estadístico realizado con el programa SPSS 19 (IBM, Armonk, New York): primero, se evaluó la distribución de los datos a través de la prueba de Kolmogorov - Smirnov, y después se aplicó el coeficiente de correlación de Spearman para las hipótesis de investigación, usando para ello un nivel de significación de 0.05 .

\section{Consideraciones éticas}

El Comité de Investigación de SES autorizó la investigación en enero del 2016. Asimismo, se sometió a revisión del Comité de Ética en Investigación de la Facultad de Medicina de la Universidad Nacional Mayor de San Marcos.

Para la participación de los trabajadores, se debió firmar previamente un consentimiento informado que explicaba el propósito de la investigación y que dejaba en claro que la participación era voluntaria y anónima.

Limitaciones, fortalezas del estudio y oportunidades de mejora

Entre las limitaciones tenemos que: No se cuenta con estudios previos que describan estas variables en la institución en otros periodos de tiempo que permitan realizar estudios comparativos.

Las fortalezas del estudio radican en que la institución estuvo dispuesta al estudio de las variables de Motivación y satisfacción pues la dirección administrativa era consciente de que permitiría cambios positivos y mejoras.

Las Oportunidades de mejora serán en el ámbito de Gestión de Recursos Humanos, pues se podrán evaluar aspectos específicos de motivación y satisfacción laboral y saber qué cambiar en la organización.

\section{RESULTADOS}

Los 136 colaboradores que participaron en la investigación pertenecen a las 7 Direcciones establecidas en el Organigrama Institucional de SES (ver Tabla 1). La mayoría estaba asignada a la Dirección de Proyectos de Investigación (DINVE) seguido por la Dirección de Proyectos de Intervención (DINTE). Según sede a la que pertenecen, la mayoría se encuentra asignado a la sede de SMP (46.3\%) seguido por la sede de Carabayllo (25.7\%).

Según sexo, la mayor parte de los encuestados es de sexo femenino (73.5\%). Según grupos etarios, la mayoría (65.4\%) tenía entre 26 y 35 años, seguido por el grupo entre los 36 y 45 años (23.5\%).

El 70.6\% de los encuestados tiene un tipo de relación contractual de plazo fijo con la institución.

La mayor proporción de los encuestados estaban trabajando de 1 a 4 años (49.3\%) y más de 5 años (27.9\%). Asimismo, los participantes estaban desempeñando su último cargo asignado por más de 1 año en un $58.8 \%$ y de 7 meses a 1 año en un $19.1 \%$.

Tabla 1. Caracterización de los colaboradores encuestados de Socios en Salud Sucursal Perú. 2016

\begin{tabular}{lcc}
\hline VARIABLE & No $^{\circ}$ & $\%$ \\
\hline Dirección a la que pertenece & 4 & 2.9 \\
Dirección General (DIGE) & 45 & 33.1 \\
Dirección de Proyectos de Investigación (DINVE) & &
\end{tabular}




\begin{tabular}{|c|c|c|}
\hline Dirección de Proyectos de Intervención (DINTE) & 39 & 28.7 \\
\hline Dirección de Laboratorio y Farmacia (DILAB) & 8 & 5.9 \\
\hline Dirección de Informática, Datos, Estadística y Sistemas (DIDES) & 12 & 8.8 \\
\hline Dirección de Desarrollo y Comunicaciones (DECO) & 4 & 2.9 \\
\hline Dirección de Administración y Finanzas (DAF) & 24 & 17.6 \\
\hline \multicolumn{3}{|l|}{ Sede a la que pertenece } \\
\hline Carabayllo & 35 & 25.7 \\
\hline ChimpuOcllo & 9 & 6.6 \\
\hline SMP* & 63 & 46.3 \\
\hline San Borja & 20 & 14.7 \\
\hline Chincha & 9 & 6.6 \\
\hline \multicolumn{3}{|l|}{ Sexo } \\
\hline Femenino & 100 & 73.5 \\
\hline Masculino & 36 & 26.5 \\
\hline \multicolumn{3}{|l|}{ Grupos etarios } \\
\hline De 18 a 25 años & 9 & 6.6 \\
\hline De 26 a 35 años & 89 & 65.4 \\
\hline De 36 a 45 años & 32 & 23.5 \\
\hline De 46 a 55 años & 5 & 3.7 \\
\hline De 56 años a más & 1 & 0.7 \\
\hline Edad media & 32.7 & (DE 6.33) \\
\hline \multicolumn{3}{|l|}{ Tipo de relación contractual } \\
\hline A plazo fijo & 96 & 70.6 \\
\hline A plazo indeterminado & 40 & 29.4 \\
\hline \multicolumn{3}{|l|}{ Tiempo de trabajo en la institución } \\
\hline De 3 a 6 meses & 15 & 11.0 \\
\hline Mayor a 6 meses pero menor a 1 año & 16 & 11.8 \\
\hline De 1 año a 4 años & 67 & 49.3 \\
\hline De 5 años a más & 38 & 27.9 \\
\hline \multicolumn{3}{|l|}{ Tiempo de trabajo en su último cargo asignado } \\
\hline Menos de 3 meses & 6 & 4.4 \\
\hline De 3 a 6 meses & 24 & 17.6 \\
\hline De 7 meses a 1 año & 26 & 19.1 \\
\hline Más de 1 año & 80 & 58.8 \\
\hline
\end{tabular}

Fuente: Encuestas aplicadas a colaboradores de SES. Febrero-Junio 2016 


\section{Motivación Laboral}

La mayor proporción del personal de SES se encuentra medianamente motivado (49.3\%), seguido por el grupo de altamente motivado (25.7\%) y bajamente motivado $(25.0 \%)$.
Se halló entonces, entre el personal de SES, una motivación global mínima de 29 puntos y máxima de 60 puntos, para una media de 49.36 (DE. 6.85).

En la Tabla 2 se presentan los promedios obtenidos para cada factor higiénico y motivacional.

Tabla 2. Motivación laboral del personal de socios en salud sucursal Perú según los factores de la Teoría Bifactorial de Frederick Herzberg. 2016

\begin{tabular}{|c|c|c|}
\hline \multirow[t]{2}{*}{ TIPO DE FACTOR } & FACTORES & PROMEDIO \\
\hline & Relaciones con el jefe & 4.21 \\
\hline \multirow{5}{*}{ Factores higiénicc } & Relaciones con los compañeros de trabajo & 4.18 \\
\hline & Buenas condiciones laborales & 4.06 \\
\hline & Seguridad de trabajo & 4.03 \\
\hline & Prestigio o status & 3.92 \\
\hline & Políticas y Directrices de la Organización & 3.88 \\
\hline \multirow{7}{*}{ Factores motivad } & El trabajo en sí mismo como estímulo positivo & 4.28 \\
\hline & responsabilidad & 4.26 \\
\hline & Autorrealización personal & 4.17 \\
\hline & & \\
\hline & Reconocimiento profesional & 4.16 \\
\hline & Oportunidad de crecimiento & 4.14 \\
\hline & Desarrollo profesional & 4.07 \\
\hline
\end{tabular}

Fuente: Encuestas aplicadas a colaboradores de SES. Febrero-junio 2016

Según los factores higiénicos, tenemos que, la mayor proporción de colaboradores se encuentra mediamente motivado (46.3\%), seguido por el grupo bajamente motivado (33.1\%) y altamente motivado (20.6\%). Los factores de mayor promedio fueron: Relaciones con el jefe y Relaciones con los compañeros de trabajo con 4.21 y 4.18 respectivamente.

Según los factores motivacionales, tenemos que, la mayor proporción de colaboradores se encuentra mediamente motivado (57.4\%), seguido por el grupo bajamente motivado (22.1\%) y altamente motivado (20.6\%). Los factores de mayores promedios fueron: El trabajo en sí mismo como Estímulo positivo y Responsabilidad con 4.28 y 4.26 respectivamente.

\section{Satisfacción Laboral}

La mayor proporción del personal de SES se encuentra medianamente satisfecho (56.6\%), seguido por el grupo de bajamente satisfecho (24.3\%) y altamente satisfecho (19.1\%).

Se halló entonces, entre el personal de SES, una satisfacción global mínima de 57 puntos y máxima de 111 puntos, para una media de 83.67 (DE. 8.96).

En la Tabla 3 se presentan los promedios obtenidos para cada componente de la Satisfacción Laboral. El componente con mayor promedio fue Relación Personal (4.32), mientras que los componentes con menor promedio fueron Presión en el trabajo (2.72) y Distensión en el trabajo (3.15). 
Tabla 3. Componentes de la satisfacción laboral del personal de socios en salud sucursal Perú, 2016

$\begin{array}{lc}\text { Componentes de Satisfacción Laboral } & \text { Promedio } \\ \text { Satisfacción en el trabajo } & 3.72 \\ \text { Presión en el trabajo } & 2.72 \\ \text { Relación personal } & 4.32 \\ \text { Distensión en el trabajo } & 3.15 \\ \text { Adecuación para el trabajo } & 3.91 \\ \text { Control sobre el trabajo } & 3.66 \\ \text { Variedad de tareas } & 3.32\end{array}$

Fuente: Encuestas aplicadas a colaboradores de SES. Febrero-junio 2016

El ítem de puntaje promedio más alto es "La relación con mis compañeros es cordial" que obtuvo 4.38 seguido por los ítems "Tengo interés por las cosas que realizo" y "La relación con mi jefe es cordial" con 4.35 y 4.27 respectivamente (Tabla 4).Por otro lado, los ítems de menor promedio son "En el trabajo diario, me tengo que emplear a fondo" (1.91), "El sueldo es adecuado" (2.61), "Al final de la jornada me encuentro muy cansado" (2.67), "No me desconecto del trabajo cuando llego a casa" (2.77) y "Me falta tiempo para realizar mi trabajo" (2.96).

Tabla 4. Satisfacción laboral del personal de socios en salud sucursal Perú según componentes, indicadores e ítems del Cuestionario FONT Roja. 2016

\begin{tabular}{|c|c|c|c|}
\hline DIMENSIÓN & INDICADOR & ITEMS & PROMEDIO \\
\hline \multirow{7}{*}{$\begin{array}{l}\text { Satisfacción } \\
\text { en el trabajo }\end{array}$} & $\begin{array}{l}\text { Satisfacción de condiciones } \\
\text { laborales }\end{array}$ & $\begin{array}{l}\text { 7. En mi trabajo, me encuentro } \\
\text { satisfecho.+ }\end{array}$ & 3.76 \\
\hline & Interés y Motivación & $\begin{array}{l}\text { 10. Tengo interés por las cosas que } \\
\text { realizo.+ }\end{array}$ & 4.35 \\
\hline & Interés y Motivación & $\begin{array}{l}\text { 11. Tengo la sensación de que lo que } \\
\text { hago no vale la pena.- }\end{array}$ & 4.01 \\
\hline & Reconocimiento & $\begin{array}{l}\text { 12. Obtengo reconocimiento por } \mathrm{mi} \\
\text { trabajo+ }\end{array}$ & 3.26 \\
\hline & Satisfacción de perfil laboral & 16. Ocupo el puesto que merezco+ & 3.57 \\
\hline & Reconocimiento & $\begin{array}{l}\text { 17. Tengo muchas posibilidades de } \\
\text { promoción+ }\end{array}$ & 3.15 \\
\hline & Satisfacción de perfil laboral & $\begin{array}{l}\text { 19. Se lo que se espera de mí en el } \\
\text { trabajo.+ }\end{array}$ & 3.96 \\
\hline
\end{tabular}




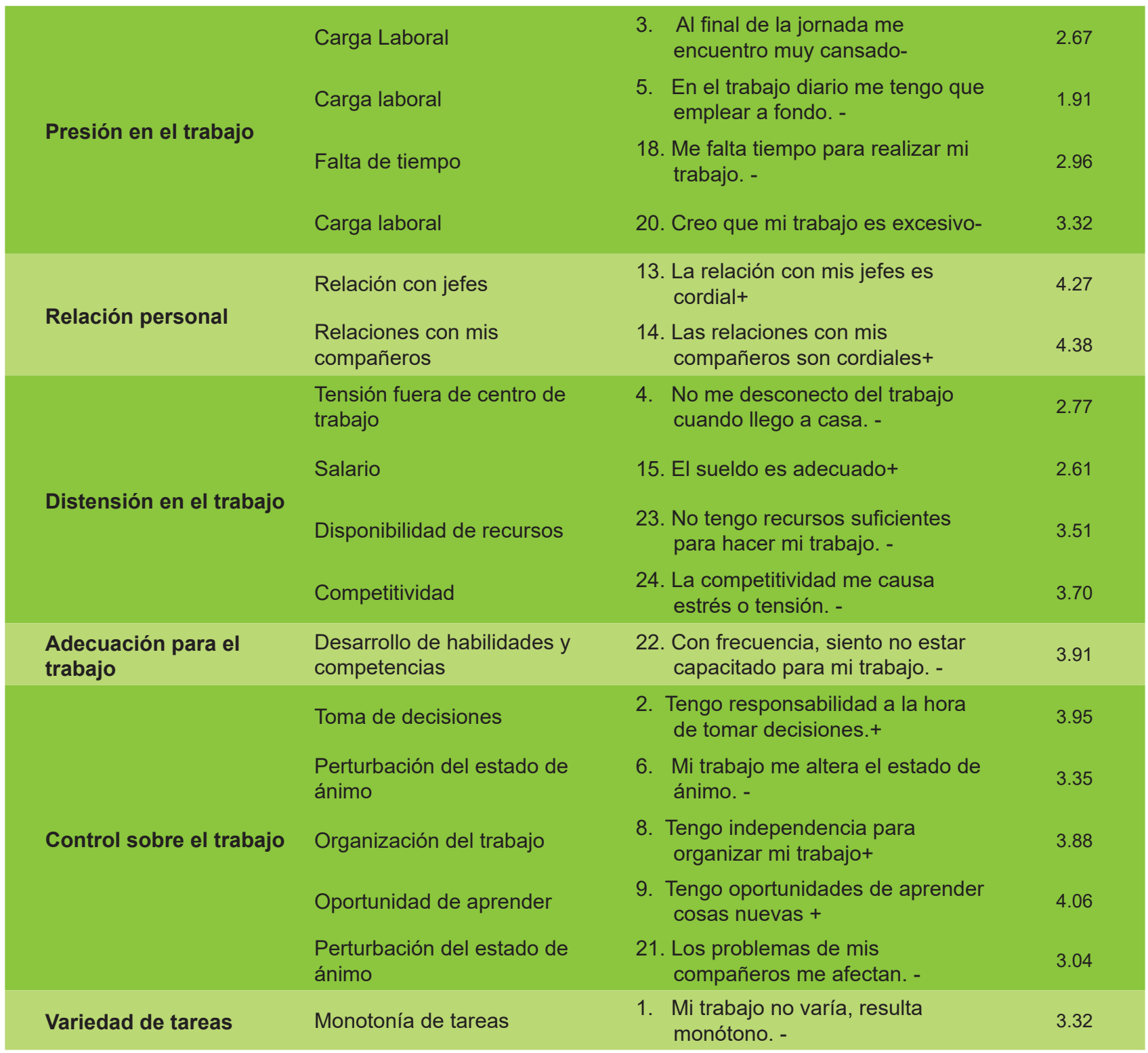

Fuente: Encuestas aplicadas a colaboradores de SES. Febrero-junio 2016

Según las indicaciones del autor, fue indispensable para el análisis e interpretación de los datos, la recodificación de todos los ítems negativos del cuestionario ya que por su contenido gramatical, la escala de Tipo Likert puntúa de forma inversa (donde valor 1 es máxima satisfacción y valor 5 es mínima satisfacción). Se aplicó la siguiente ecuación de densidad de cambio:

$Y$ (nuevo valor asignado $)=6-x$ (valor marcado por el participante durante la aplicación del cuestionario)

\section{Relación entre motivación y satisfacción laboral}

La prueba de Kolmogorov - Smirnov mostró que los datos presentaban distribución no normal por lo cual, para determinar la relación estadística entre las dos variables, se usaron los coeficientes de correlación de Spearman.

Dado que $p<0.001$, se rechaza la hipótesis nula "HO: Existe correlación nula y no significativa entre motivación laboral y la satisfacción laboral del personal de Socios en Salud Sucursal Perú" (Ver Tabla 5).Por tanto, se determina que existe correlación positiva $(0.336)$ entre ambas variables aunque de baja intensidad, lo cual nos permite aseverar que A mayor grado de motivación laboral, mayor grado de satisfacción laboral del personal de Socios en Salud Sucursal Perú. 
Tabla 5. Estadístico de prueba RHO de Spearman entre motivación y satisfacción laboral del personal de socios en salud sucursal Perú

\begin{tabular}{|c|c|c|c|}
\hline & & \multicolumn{2}{|c|}{ Satisfacción Laboral } \\
\hline \multirow[t]{3}{*}{ Rho de Spearman } & Motivación Laboral & Coeficiente de correlación & $0,336^{* *}$ \\
\hline & & Sig. (bilateral) - valor $p$ & 0,000064 \\
\hline & & $\mathrm{N}$ & 136 \\
\hline
\end{tabular}

Fuente: Análisis estadístico de los datos recolectados con paquete estadístico SPSS

La Figura 1 muestra el gráfico de dispersión de los datos. Si trazamos una línea se puede visualizar una tendencia de correlación lineal positiva y significativa entre la motivación y la satisfacción laboral, es decir, a cuanto mayor es la motivación laboral de los trabajadores, más satisfechos se encontrarán los trabajadores.

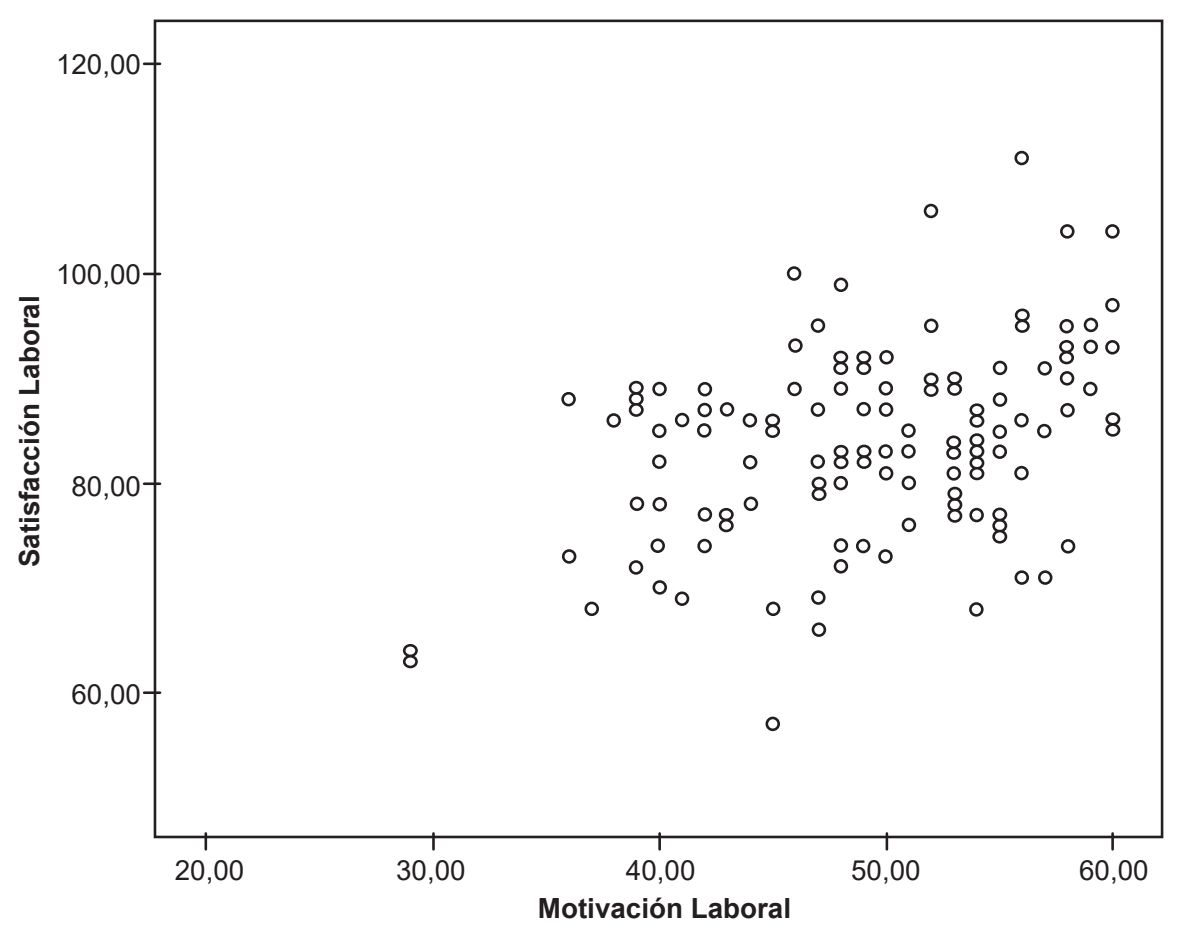

Figura 1. Dispersión entre la motivación laboral y satisfacción laboral del personal de socios en salud sucursal Perú, 2016

Para las hipótesis específicas, también se procedió a hallar el coeficiente de correlación de Spearman:

- Dado que $p<0.001$, se rechaza la hipótesis nula para la hipótesis específica 1. Por tanto, se determinó una baja correlación positiva $(0,299)$ que permite aseverar que A mayor grado de motivación laboral en los factores higiénicos, mayor grado de satisfacción laboral del personal de Socios en Salud Sucursal Perú.

- Dado que $p<0.001$, se rechaza la hipótesis nula para la hipótesis específica 2. Por tanto, se determinó una baja correlación positiva $(0.346)$ que permite aseverar que A mayor grado de motivación laboral en los factores motivacionales, mayor grado de satisfacción laboral del personal de Socios en Salud Sucursal Perú.

\section{DISCUSIÓN}

Referente a la motivación laboral, una cuarta parte de los trabajadores se encuentran altamente motivados y de acuerdo a la literatura, se espera que tengan un mayor rendimiento y desempeño laboral ${ }^{(18)}$. Para mejorar la motivación laboral de los colaboradores que se encuentran "medianamente motivados" y "bajamente motivados", se puede sugerir el enriquecimiento de trabajo que permitirá otorgar mayores responsabilidades al empleado, dando tareas cada vez más complejas de manera que este encuentre la oportunidad de satisfacer sus necesidades de crecimiento personal. Para esto, Herzberg incluso, señala 7 estrategias: eliminación de controles innecesarios, aumento de la responsabilidad, asignación 
de los empleados a unidades naturales y completas de trabajo (sección, división, etc), conceder más autonomía en las actividades, elaboración de informes periódicos sobre calidad de desempeño, introducción de nuevas e interesantes tareas y asignación a tareas especializadas ${ }^{(19)}$.

Según Herzberg, los factores higiénicos están relacionados a las condiciones que la empresa puede administrar. Considerando solo los factores higiénicos, un tercio de los trabajadores se encuentra bajamente motivado, lo que manifiesta la necesidad de que la organización redireccione sus esfuerzos y aplique nuevas estrategias para reducir la brecha existente, que permitiría incrementar el compromiso, alinear las metas individuales con las organizacionales y facilitar el camino hacia el alcance de metas institucionales.

Se puede decir que los factores higiénicos que tienen un promedio superior a 4 son adecuadamente considerados, trabajados y/o priorizados en la organización, los cercanos a 3 denotan indiferencia y los resultados de 1 y 2 son negativos para la motivación. El 33\% (2 de 6) de los factores higiénicos tiene un promedio que en la escala es de 3 , indiferente, que corresponde a los aspectos relacionados con "Prestigio o status" y "Políticas y directrices de la organización".

Según Herzberg, los factores motivacionales están relacionados directamente a la persona. Considerando solo los factores motivacionales, la quinta parte de los trabajadores se encuentra bajamente motivado lo que muestra la necesidad de sumar esfuerzos y modificar estrategias para disminuir esta proporción.

Usando la premisa de que los factores que tienen un promedio superior a 4 son adecuadamente considerados, trabajados y/o priorizados en el entorno de la organización, el $100 \%$ (6 de 6) de los factores motivacionales superan los 4 puntos de promedio, lo que indica que el trabajo desarrollado es bueno para la motivación laboral de los trabajadores. Además, ya que las características de los puestos de trabajo de la organización permiten tener un estímulo positivo para las personas, habrían altas posibilidades de que el nivel de motivación mejore si la organización trabajara tomara en cuenta los hallazgos de esta investigación.

Chang en su investigación, encuentra que el 95.2\% de médicos del Hospital Nacional Arzobispo Loayza tuvo una motivación alta mientras que $4.8 \%$ tuvo una motivación media y ninguno tuvo motivación baja. Es evidente que estos resultados discrepan y probablemente se debe a que la medición de la variable se hizo con otro instrumento y los participantes del estudio eran sólo personal médico ${ }^{(20)}$. Aún así, se podría esperar mayor motivación de una organización del Sector Privado que en una institución pública; sin embargo este resultado podría deberse a que el Hospital Nacional Arzobispo Loayza es uno de los hospitales de mayor complejidad en el Perú y por ello, los factores motivadores podrían tener mayor relevancia que los factores higiénicos.

Del Río-Mendoza y colaboradores, al analizar el clima organizacional en trabajadores de un Hospital General de
Ica en 2013, encontraron que la motivación connotaba un "clima por mejorar", lo que mostraría un aspecto que requeriría un proceso de mejora. Esta situación es similar a la de SES, donde hay aspectos que deben mejorarse para incrementar la proporción de colaboradores altamente motivados $^{(21)}$.

En el caso de la satisfacción laboral, las tres cuartas partes de los trabajadores se encuentran en las categorías de altamente satisfecho y medianamente satisfecho lo que indica que hay un estado positivo de la percepción subjetiva de sus experiencias laborales. Esto tendría un alto impacto personal y organizacional pues se espera tener buena calidad de vida y bajas tasas de accidentabilidad, ausentismo y abandono de la organización ${ }^{(22)}$.

El componente con promedio más alto fue "Relación Personal" mientras que, los componentes a los que se les debe dar prioridad por sus bajos promedios globales son: "Presión en el trabajo", "Variedad de la tarea"y "Distensión en el trabajo”.

Cifuentes y Manrique evaluaron la satisfacción laboral en un institución de salud de cuarto nivel de atención en Bogotá, Colombia usando el instrumento FONT Roja, hallando una satisfacción laboral global mínima de 56 puntos y máxima de 82 puntos, con una media de 69.98 (DE: 5.67). Estos resultados son menores a los puntajes que obtuvimos pues nuestra media es de 83.67 puntos (valor mínimo de 57 y valor máximo de 111 puntos); sin embargo los resultados de Cifuentes presentan una menor desviación estándar respecto a nuestra muestra (8.96). Por otro lado, Cifuentes encontró que solo las características extrínsecas del estatus, promoción profesional, distensión relacionada con el trabajo y relación interpersonal con los jefes $(3.34,3.33$, 3.16 y 3.01 respectivamente) tuvieron una media superior a los 3 puntos. Esto discrepa con nuestro hallazgo, en el que 6 componentes superaron la media de 3 puntos y sólo el componente Presión en el trabajo tuvo $2.72^{(23)}$.

Vera-Martínez y colaboradores aplicaron el cuestionario en el personal sanitario de prisiones españolas, y obtuvieron bajas puntuaciones en las dimensiones de Variedad de la Tarea (media 1.66), Presión en el Trabajo (media 2.15) y Control sobre el trabajo (media 2.77). Respecto a nuestro estudio, se coincide en que la dimensión Presión en el Trabajo alcanza un promedio muy bajo ${ }^{(24)}$.

Como se ha visto, el nivel de la satisfacción laboral varía de una institución a otra debido a que es condicionado a las características propias de la organización y a su clima organizacional.

Referente a la relación entre la motivación y satisfacción laboral, Castillo establece una correlación significativa, positiva y mediana entre las variables de clima organizacional, motivación intrínseca y satisfacción laboral; lo que coincide con el hallazgo de esta investigación (existe correlación entre las variables de motivación y satisfacción laboral) en términos de dirección (ambos la reportan como positiva) pero no en términos de intensidad, pues Castillo reporta 
que la correlación es significativa y mediana mientras que, esta investigación establece una correlación positiva baja. Entonces, Castillo concluye que, al elevarse la percepción favorable del clima laboral y al reportar mayor motivación intrínseca, la satisfacción laboral será más elevada ${ }^{(25)}$. Por el contrario a nuestros hallazgos, Baldeón-Trejo en el 2015 no encontró asociación significativa entre la motivación y la satisfacción laboral ${ }^{(26)}$.

Briceño en su investigación en el Hospital Nacional Santa Rosa, analiza el nivel de motivación y su relación con la satisfacción laboral del profesional de enfermería en el Área de Centro Quirúrgico. El autor concluye que el $43.5 \%$ tienen motivación media, $34.8 \%$ alta y $21.7 \%$ baja y sobre la satisfacción laboral, $43.5 \%$ presentan satisfacción media, $34.8 \%$ alta y $22.7 \%$ baja, y además acepta que existe relación entre el nivel de motivación y satisfacción laboral usando la correlación de Spearman. Los resultados tienen una distribución similar a la hallada en esta investigación ya que la mayor proporción se concentra en el valor "medianamente motivado" y "medianamente satisfecho" (27).

Vásquez también analizó las dos variables, y concuerda con nuestros resultados, donde se obtuvo que el $48 \%$ de profesionales de enfermería en el Hospital Nacional Arzobispo Loayza tenía motivación media, 27\% alta y 25\% baja; mientras que $53.8 \%$ tenía satisfacción laboral medio, $21.2 \%$ bajo y $25.0 \%$ alto. Su conclusión, igual que la nuestra, es que existe relación entre el nivel de motivación y la satisfacción laboral, el cual influirá en el desempeño de los profesionales así como en la calidad de atención ${ }^{(28)}$.

La relación motivación-satisfacción laboral que encuentra Sánchez Reynalte en los colaboradores administrativos de una red de salud de la ciudad de Chimbote es descrita como una correlación muy alta y significativa pues tuvo un coeficiente de correlación de Pearson de $0.887^{(29)}$. Esto discrepa con nuestro hallazgo en cuanto a la intensidad de la correlación entre variables.

En conclusión, con nuestra investigación se acepta que "A mayor grado de motivación laboral, mayor grado de satisfacción laboral del personal de Socios en Salud Sucursal Perú". La relación entre la motivación y satisfacción laboral es de correlación positiva aunque de baja intensidad. Asimismo se aceptaron las dos hipótesis específicas propuestas: “A mayor grado de motivación laboral en los factores higiénicos, mayor grado de satisfacción laboral del personal de Socios en Salud Sucursal Perú" y "A mayor grado de motivación laboral en los factores motivacionales, mayor grado de satisfacción laboral del personal de Socios en Salud Sucursal Perú".

El nivel de la motivación laboral es medianamente motivado. El nivel de la satisfacción laboral es medianamente satisfecho.

Sería pertinente y oportuno que los hallazgos de la investigación sean considerados (aspectos que presentaron menores promedios) para la mejora de aspectos de la Gestión de Recursos Humanos.
AGRADECIMIENTO: A la Dirección General y a la Dirección Administrativa de Socios en Salud Sucursal Perú por brindar facilidades para la ejecución de la investigación.

\section{REFERENCIAS BIBLIOGRÁFICAS}

1. Socios en Salud Sucursal Perú.[Internet].Lima, 2017. [citada 18 de agosto del 2017] Disponible en: https://sociosensalud. org.pe/nuestro-trabajo/.

2. Socios en Salud Sucursal Perú. [Internet]Lima, 2014. Manual de Políticas. 2014. [citada 18 de agosto del 2017]Disponible en: http://intranet. sociosensalud.org.pe:8080/intranet/wpcontent/uploads/2017/09/MANUAL_POLITICAS_2014.pdf.

3. Socios en Salud Sucursal Perú. [Internet]Lima, 2014. Plan Estratégico 2014-2019. [citada 18 de agosto del 2017] Disponible en: http://intranet. sociosensalud.org.pe:8080/intranet/wpcontent/uploads/2017/09/PLAN_ESTRATEGICO_2014_2019.pdf.

4. Arias F, Heredia V. Administración de recursos humanos para el alto desempeño. 5a ed. México DF: Trillas; 2006

5. Vadillo M. Liderazgo y motivación de equipos de trabajo. 8a ed. Mardrid:ESIC Editorial. 2013.

6. Cerón J. La importancia de la motivación en las empresas. [Tesis de bachiller]. Bogota:Universidad Militar Nueva Granada; 2016

7. Scott W, Davis G. Organizations and organizing: Rational, natural and open systems perspectives. 1a ed. Nueva York: Routledge. 2015.

8. Buitrago J. La motivación y el liderazgo claves del Éxito en la Policía Nacional [Tesis de Bachiller]. Bogota:Universidad Militar Nueva Granada;2017

9. Herzberg F, Mausner B, Snyderman B. The Motivation to Work. 1a ed. Estados unidos: Transaction publishers; 2011

10. Herzberg F. The Motivation-Hygiene Concept and Psycotherapy. Personnel Administration. 1964

11. Olarte D. La motivación como eje principal en el incremento de la productividad laboral en las organizaciones colombianas. [Tesis de Bachiller]. Bogota:Universidad Militar Nueva Granada. 2017

12. Correa F. Los factores higiénicos de Frederick Herzberg no son suficientes para motivar. [Tesis de Bachiller]. Mar de Plata:Universidad FASTA; 2012.

13. Bilbao J, Vega M. NTP 394: Satisfacción laboral: Escala General de Satisfacción. España: Ministerio de Trabajo y Asuntos Sociales. Instituto Nacional de Seguridad e Higiene Personal; 2010

14. Bustamante E. El clima de comunicación, la motivación y la satisfacción laboral en un proceso de atención primaria en Colombia. Rev Comunicación y Salud. 2013; 3 (1): 35-49.

15. Patlán-Pérez J, Martínez E, Hernández R. El Clima y la Justicia Organizacional y su Efecto en la Satisfacción Laboral. Revista Internacional Administración y Finanzas. 2012; 5(5): 1-19

16. Orgambídez-Ramos A, Pérez-Moreno P, Borrego-Alés Y. Estrés de rol y satisfacción laboral: examinando el papel mediador del engagement en el trabajo. Rev Psicol Trab Org. 2015; 31(2), 69-77

17. Kar F, Bin M, Sorooshian S, Bin S. Implementation Of Total Quality Management And ISO 9000 Certification. International Journal of Engineering Applied Sciences and Technology. 2016; 1 (6), 33-6.

18. Marshall S. Dirigir Motivando: manual del monitor y cuestionario MBM. 3a ed. Madrid: Ramon Areces. 1998

19. Manso J. El legado de Frederick Irving Herzberg. Revista Universidad EAFIT. 2012;8(128): 78-86.

20. Chang A. Estudio de la motivación laboral y el conocimiento de la necesidad predominante según la teoría de las necesidades de McClelland, en los médicos del Hospital Nacional Arzobispo Loayza. [Tesis]. Lima: Universidad Nacional Mayor de San Marcos; 2010 
21. Del Rio-Mendoza J, Munares-Lovaton A, Montalvo-Valdez H. Clima organizacional en trabajadores de un hospital general de Ica, Perú. Rev Méd Panacea. 2013; 3(1): 11-4.

22. Mendoza-Llanos R. Calidad de vida y satisfacción laboral en trabajadores y trabajadoras del sector comercial de la ciudad de Chillán. [Tesis]. Chillán:Universidad Del Bio-Bio; 2015

23. Cifuentes J, Manrique F. Satisfacción laboral en enfermería en una institución de salud de cuarto nivel de atención, Bogotá, Colombia. Av Enferm, 2014; 32(2): 217-27

24. Vera-Remartínez E, Mora L, Gonzales J, García J, Garcés E, Domínguez J, et al. Satisfacción laboral de la enfermera en las prisiones españolas. Rev Esp Sanid Penit. 2009; 11 (3): 80-6

25. Castillo N. Clima, motivación intrínseca y satisfacción laboral en trabajadores de diferentes niveles jerárquicos. [Tesis]. Lima: Pontificia Universidad Católica del Perú; 2014

26. Baldeón C. Factores Asociados a la Satisfacción del Personal de Salud del Hospital IV Ramiro Priale Priale, Huancayo. Ágora Rev Cient. 2015; 2(1), 112-24.

27. Briceño C, Del Rosario Y. Nivel de motivación y su relación con la satisfacción laboral del profesional de enfermería en el Área de Centro Quirúrgico del Hospital Nacional Santa Rosa: [Disertación]. Lima: Universidad Nacional Mayor de San Marcos. Facultad de Medicina Humana; 2010.

28. Vásquez S. Nivel de motivación y su relación con las satisfacción laboral del profesional de enfermería en el Hospital Nacional Arzobispo Loayza, 2006. [Tesis]. Lima: Universidad Nacional Mayor de San Marcos; 2007.

29. Sánchez R. Motivación y Satisfacción Laboral en los colaboradores administrativos de una red de salud de la ciudad de Chimbote. [Tesis]. Chimbote: Universidad Cesar Vallejo; 2016.

\section{Fuentes de Financiamiento}

Este artículo ha sido financiado por los autores.

\section{Conflicto de interés}

HSMS es Coordinadora de Proyecto de la Dirección de Proyectos de Investigación de Socios en Salud Sucursal Perú.

MDPM declara no tener ningún conflicto de interés

\section{Correspondencia:}

Helen Stephani Marin Samanez

Dirección: Socios en Salud Sucursal Perú.

Av. Túpac Amaru 4480. Comas. Lima, Perú

Teléfono: 942603852

Correo electrónico: estimsanmartin@gmail.com

Recibido: 24 de agosto de 2017

Evaluado: 27 de agosto de 2017

Aprobado: 06 de octubre de 2017

(c) La revista. Publicado por Universidad de San Martín de Porres, Perú. (cc) Br Licencia de Creative Commons Artículo en acceso abierto bajo términos de Licencia Creative Commons Atribución 4.0 Internacional. (http://creativecommons.org/licenses/by/4.0/) 